\title{
A FINITE ELEMENT DEVELOPMENT FOR BALL BEARING NONLINEAR STIFFNESS MODELIZATION
}

\author{
Hentati, T.; Dammak, F.; Fakhfakh, T. \& Haddar, M. \\ Mechanics Modeling and Production Research Unit, Mechanical Engineering Department \\ National School of Engineers of Sfax (ENIS), BP. W. 3038 Sfax, Tunisia \\ E-mail: mohamed.haddar@enis.rnu.tn
}

\begin{abstract}
A formulation and algorithmic treatment of a three-dimensional bearing stiffness is presented. This stiffness formulation is based on the non-linear equilibrium balance of forces and moments on the rolling elements, in a ball bearing, exerted by the inner and the outer races including contact forces. Newton-Raphson Method is used to solve the resulting non linear equation system. A non-linear two nodes finite element linking up a node of the inner race to another node of the outer race is developed. The tangent stiffness matrix of this element is deduced from the Jacobean of the convergent equilibrium. A numerical study is presented showing the influence of some parameters variation and the coupling between all stiffness terms.

(Accepted by previous Editorial Team.)
\end{abstract}

Key Words: Ball Bearing, Finite Element Method, Stiffness Matrix, Newton-Raphson Method

\section{INTRODUCTION}

The rolling bearings are used to perform a pivot link between two sets of pieces of a mechanism. The rolling bearing is made up of an outer race, an inner race and rolling elements located in the appropriate cage. The large use of rolling bearings proves their necessity in the modern industries.

As computer technology progresses, computer modelization becomes popular and essential to shorten the lead-time and product development. Rolling bearings designers use more and more computer to design appropriate bearings for user requirements within a short time. Static and dynamic analyses of rolling bearings with computer are very popular. Static analysis, which will be considered in this paper, is important for the bearing designers to know basic information such as the internal load distribution.

The rolling bearings was generally considered as system boundary conditions (hinge, clamped) or simply modelled by the association of springs in the axial and the radial directions. Jones [1] and Palmgren [2] are the first authors who have developed a simple analytic model of a rolling bearing loaded in the radial or axial direction using the Hertz and Boussineq theories. While [3] has modelled the roller bearing by axial and radial stiffnesses in the ball bearing and cylindrical roller bearing. Simple formulas presented by Garguillo can determinate those stiffnesses in some configurations. Krauss [4] showed the influence of some experimental parameters (rotating velocity, contact angle, damper ...) on the rolling bearings stiffnesses. Drago [5] defined angular stiffness in the shaft flexion plan. Lim and Singh [6] suggested an analytic approach based on the determination of a stiffness matrix associated to five degrees of freedom of the inner race (three translations and two rotations). Demul [7] used this model and add to it the centrifugal forces. Bourdon [8] and Lahmar [9] used the approach proposed by Singh and inject the resulting stiffness matrix in the global system studied. Gupta [10] proposed an analytic formulation of all rolling bearing parts and determined the geometric interaction between all terms in the rolling bearing. Nelias [11] 
developed a model where he considered the cage and the lubricant film on all contacts in the rolling bearing equilibrium.

In this paper, we present a formulation and algorithmic treatment of a three-dimensional bearing stiffness. This formulation is base on the non-linear equilibrium of forces and moments exerted by the inner and the outer races on the rolling elements by means of the Hertz theory. The resulting non linear equation system is solved using the Newton-Raphson Method. A non-linear two nodes finite element linking up a node of the inner race to another node of the outer race is developed. The tangent stiffness matrix of this element is deduced from the Jacobean of the convergent equilibrium of forces and moments. The stress distribution on the rolling elements and the races are determined. The influence of the variation of some parameters such as the angular position of the rolling element and the contact angle on the stiffness matrix coefficients is studied. The important coupling, neglected until now, between the stiffness matrix coefficients are demonstrated.

\section{THEORETICAL MODEL}

The main idea of our model is to partition the rolling bearing in rolling elements (ball or roller) and associate to every rolling element a finite element. This element (rolling element), link up one node of the inner race $N_{2}$ to another node of the outer race $N_{1}$ by mean of the stiffness matrix. This matrix transmits three forces and two moments. So, it is necessary to determinate for every rolling element and for given displacements of the two nodes $N_{l}$ and $N_{2}$, the displacement of the rolling element center for which the equilibrium is reached.

The two races (inner and outer) displacements caused forces on the bearing trail and on the race flange. These forces are estimated by the Hertz theory

$$
Q=C_{f}(\delta)^{n}
$$

where:

$Q \quad$ - force exerted on the rolling element,

$C_{f} \quad$ - stiffness constant depending the nature of the material,

$\Delta \quad$ - geometric interaction between the two bodies,

$n \quad$ - coefficient depending on the contact nature.

Three coordinate systems are used in this study given by Fig. 1:

$R_{1}\left(O, \overrightarrow{e_{x}}, \overrightarrow{e_{y}}, \overrightarrow{e_{z}}\right)$ global coordinate system associated to the structure,

$R_{2}\left(C_{r}, \overrightarrow{e_{r}}, \overrightarrow{e_{n}}, \overrightarrow{e_{z}}\right)$ coordinate system associated to the rolling element,

$R_{3}\left(C_{r}, \overrightarrow{e_{r^{\prime}}}, \overrightarrow{e_{n}}, \overrightarrow{e_{z^{\prime}}}\right)$ coordinate system associated to the outer race.

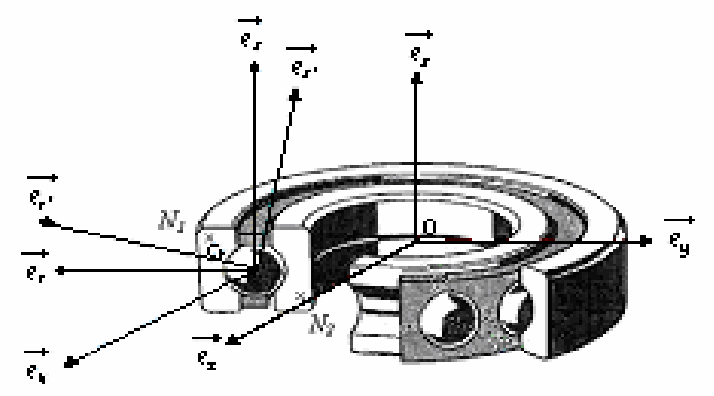

Figure 1: The coordinate systems used in this study. 
We define $\theta_{x}, \theta_{y}$ and $\theta_{z}$ the angular displacements of the node $N_{l}$ calculated in $R_{l}$ and $\gamma$ the angular deviation of the outer race in $R_{2}$ which can be written as

$$
\gamma=-\sin \phi \theta_{x}+\cos \phi \theta_{\mathrm{y}}
$$

with $\phi$ the angle defining the position of the rolling element center $C_{r}$.

The "Geometry matrixes" $\left[G_{1}\right]$ and $\left[G_{2}\right]$ which correspond respectively to the outer and inner races are given by equation (3). Those matrixes allow transposing the node $N_{i}$ displacement to the rolling element center displacement.

$$
\left.\left.\left\{\overrightarrow{\operatorname{dep}}\left(C_{r} \in(i) \text { race }\right)\right\}_{R_{3}}=\left[G_{i}\right]\right\} \overrightarrow{d e p}\left(N_{i} \in(i) \text { race }\right)\right\}_{R_{1}}
$$

We can write:

$$
\left[G_{i}\right]=\left[\begin{array}{cccccc}
\cos \gamma \cos \phi & \cos \gamma \sin \phi & \sin \gamma & -Z_{i} \sin \phi & Z_{i} \cos \phi & 0 \\
-\sin \gamma \sin \phi & \sin \gamma \sin \phi & \cos \gamma & R_{i} \sin \phi & -R_{i} \cos \phi & 0 \\
0 & 0 & 0 & -\sin \phi & \cos \phi & 0
\end{array}\right]
$$

where $Z_{i}$ and $R_{i}$ are the coordinates of the node $N_{i}$ in $R_{3}$.

The inner race displacement vector in $R_{3}$ can be written as

$$
\{\vec{u}\}_{R_{3}}=\left\{\begin{array}{l}
u_{r} \\
u_{z} \\
u_{\theta}
\end{array}\right\}=\left[G_{2}\right]\left(\left\{\overrightarrow{\operatorname{dep}} N_{2}\right\}_{R_{1}}-\left\{\overrightarrow{\operatorname{dep}} N_{1}\right\}_{R_{1}}\right)
$$

and the rolling element center displacement vector can be written as

$$
\{\vec{v}\}_{R_{3}}=\left\{\begin{array}{c}
v_{r} \\
v_{z} \\
v_{\theta}
\end{array}\right\}
$$

The residual force vector, which consists on the balance of forces and moments on the rolling element, can be written as the sum of the forces exerted by the inner and the outer races:

$$
\left\{\begin{array}{l}
\mathrm{r} \\
R
\end{array}\right\}=\sum F_{\text {ext }}=\left\{\begin{array}{l}
F_{r}^{O R} \\
F_{z}^{O R} \\
M_{\theta}^{O R}
\end{array}\right\}+\left\{\begin{array}{l}
F_{r}^{I R} \\
F_{z}^{I R} \\
M_{\theta}^{I R}
\end{array}\right\}=\left\{\begin{array}{l}
\mathrm{r} \\
\left.R\left(v_{r}, v_{z}, v_{\theta}\right)\right\}
\end{array}\right.
$$

These three non linear equations, functions of the rolling element center displacement, are solved simultaneously using the Newton-Raphson method. This method is summarized by the following system resolution and updating at each iteration:

$$
\left\{\begin{array}{c}
{\left[K_{T}\left(v^{i}\right)\right]\left\{\Delta v^{i}\right\}=-\left\{R\left(v^{i}\right)\right\}} \\
\left\{v^{i+1}\right\}=\left\{v^{i}\right\}+\left\{\Delta v^{i}\right\}
\end{array}\right.
$$

The process consists in determining, for every iteration, the residual force vector $\{\vec{R}\}$ and the Jacobean matrix $\left[K_{T}\right]$ which are function of the new displacement vector of the rolling 
element center. The Jacobean matrix $\left[K_{T}\right]$ is decomposed, as the residual vector $\{\vec{R}\}$ (eq. 7), in two parts:

$$
\left[K_{T}\right]=\left[K_{T}^{O R}\right]+\left[K_{T}^{I R}\right]
$$

where $\left[K_{T}^{O R}\right]$ is the Jacobean matrix which results from the contribution of the outer race forces and thus takes the form:

$$
\left[K_{T}^{O R}\right]=\left[\frac{\partial\left\{\stackrel{r}{F}^{O R}\right\}}{\partial\{\stackrel{\mathrm{r}}{V}\}}\right]=\left[\begin{array}{ccc}
\frac{\partial F_{r}^{O R}}{\partial v_{r}} & \frac{\partial F_{r}^{O R}}{\partial v_{z}} & \frac{\partial F_{r}^{O R}}{\partial v_{\theta}} \\
\frac{\partial F_{z}^{O R}}{\partial v_{r}} & \frac{\partial F_{z}^{O R}}{\partial v_{z}} & \frac{\partial F_{z}^{O R}}{\partial v_{\theta}} \\
\frac{\partial M_{\theta}^{O R}}{\partial v_{r}} & \frac{\partial M_{\theta}^{O R}}{\partial v_{z}} & \frac{\partial M_{\theta}^{O R}}{\partial v_{\theta}}
\end{array}\right]
$$

and $\left[K_{T}^{I R}\right]$ is the Jacobean matrix which results from the contribution of the inner race forces and thus takes the form:

$$
\left[K_{T}^{I R}\right]=\left[\frac{\partial\left\{\stackrel{r}{F}^{I R}\right\}}{\partial\{\stackrel{\mathrm{r}}{V}\}}\right]=\left[\begin{array}{ccc}
\frac{\partial F_{r}^{I R}}{\partial v_{r}} & \frac{\partial F_{r}^{I R}}{\partial v_{z}} & \frac{\partial F_{r}^{I R}}{\partial v_{\theta}} \\
\frac{\partial F_{z}^{I R}}{\partial v_{r}} & \frac{\partial F_{z}^{I R}}{\partial v_{z}} & \frac{\partial F_{z}^{I R}}{\partial v_{\theta}} \\
\frac{\partial M_{\theta}^{I R}}{\partial v_{r}} & \frac{\partial M_{\theta}^{I R}}{\partial v_{z}} & \frac{\partial M_{\theta}^{I R}}{\partial v_{\theta}}
\end{array}\right]
$$

The residual force vector is null, since the equilibrium is reached. The tangent stiffness matrix associated to the rolling element is written in referential $R_{l}$ as following:

$$
\left[K^{e}\right]_{(12 \times 12)}=[G]^{t}[\widetilde{K}][G]
$$

where the matrix $[\widetilde{K}]$ is given by the following expressions:

and

$$
[\widetilde{K}]_{(6 \times 6)}=\left[\begin{array}{cc}
K & -K \\
-K & K
\end{array}\right], \quad[K]=\left[K_{T}^{O R}\right]\left[K_{T}^{O R}+K_{T}^{I R}\right]^{-1}\left[K_{T}^{I R}\right]
$$

$$
[G]=\left[\begin{array}{rr}
G_{1} & 0 \\
0 & G_{2}
\end{array}\right]
$$
$R_{1}$ :

The forces exerted by the rolling element on the outer and the inner race can be written in

$$
\begin{aligned}
& {\left[F^{N_{1}}\right]_{R_{1}}=\left[G_{1}\right]^{t}\left[F^{O R}\right]} \\
& {\left[F^{N_{2}}\right]_{R_{1}}=\left[G_{2}\right]^{t}\left[F^{I R}\right]}
\end{aligned}
$$

The schematic layout describing the calculation steps is presented in Fig. 2. 


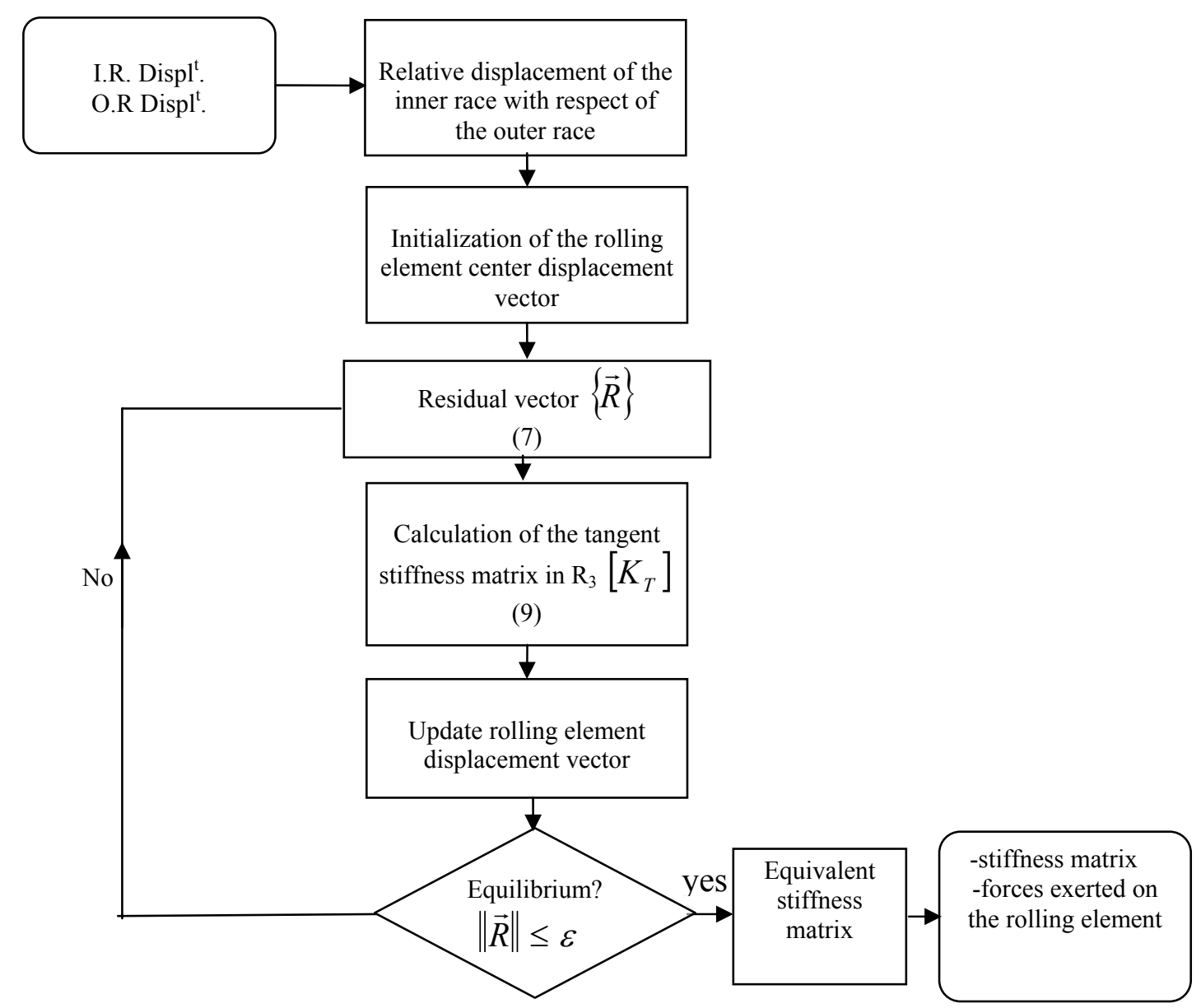

Figure 2: Algorithm of non linear equilibrium resolution.

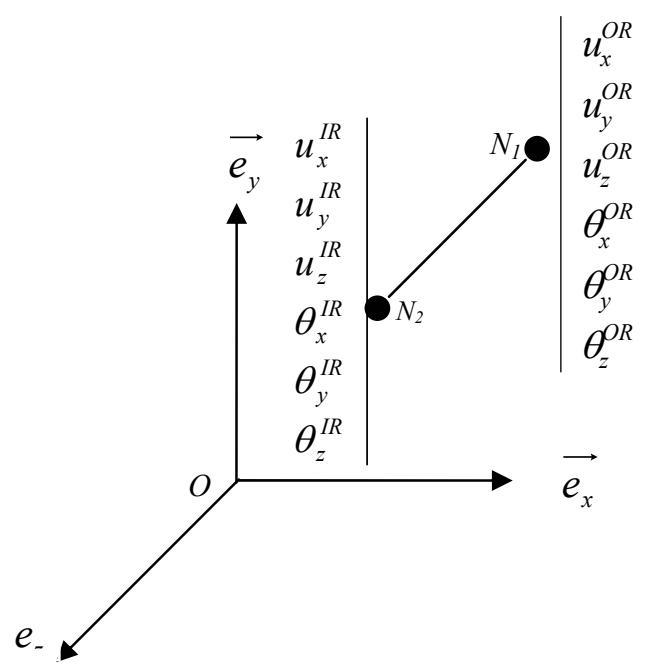

Figure 3: Finite element developed.

A finite element (figure 3) linking up one node of the inner race to another node of the outer race having six degrees of freedom by node, is developed.

The tangent stiffness matrix, of this two nodes element is based on equation (12). 


\section{BALL BEARING}

The theoretical model developed in section 2 is valid for a large kind of rolling bearing. In this section, we adopt this theoretical model for the ball bearing presented in Fig 4.

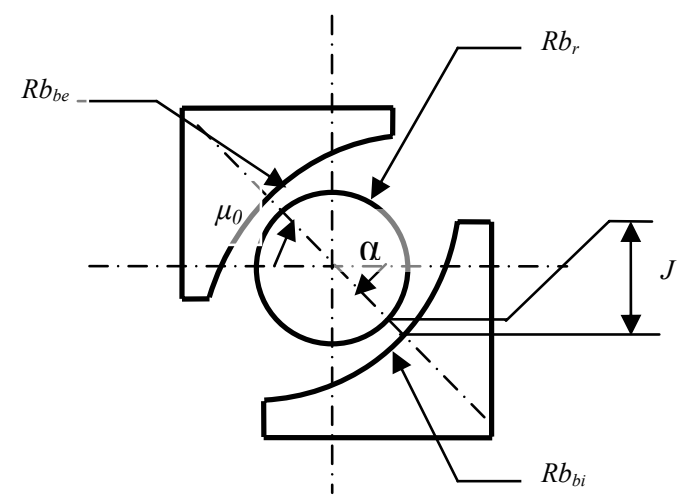

Figure 4: The ball bearing used in the application.

$$
\begin{aligned}
& \mu_{0}=90-\alpha, \quad \alpha-\text { the contact angle } \\
& R_{b r} \text { - the ball radius, } \\
& R b_{b e} \text { - the radius of curvature of the outer race groove, } \\
& R b_{b i} \text { - the radius of curvature of the inner race groove, } \\
& J \text { - the initial gap between the races and the ball. }
\end{aligned}
$$

In order to determinate the tangent stiffness matrix, we must compute the contact deflections and then the forces exerted by the inner and the outer races using the Hertz theory.

The case of shaft rotating is used here. The angular displacement $u_{\theta}$ of the inner race is the angle between the outer race axis and the inner race axis (Fig. 5):

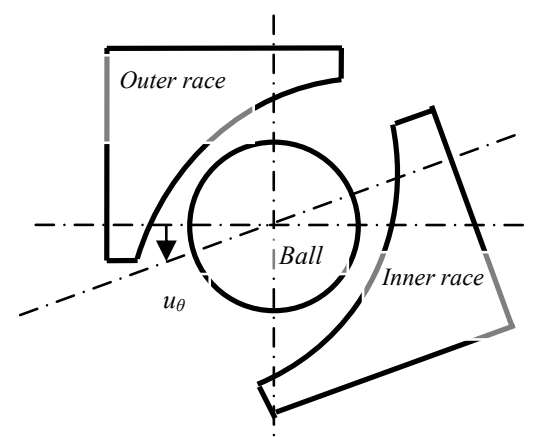

Figure 5: Angle locating the inner race with respect to the outer race.
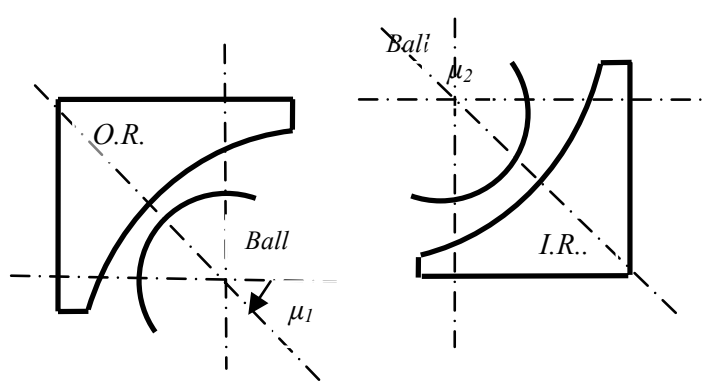

Figure 6: Contact directions. 
Fig. 6 represents the angles $\mu_{1}$ and $\mu_{2}$ (angle between the ball axis and the perpendicular to the contact) associated respectively to the effective ball direction with respect to the outer race and the inner race.

$$
\mu_{1}=\mu_{0} \text { and } \mu_{2}=\mu_{0}+u_{\theta}
$$

The gap between the ball and the races can be written as

$$
\widetilde{J}=J+R_{b r}\left(\cos \mu_{i}-\cos \mu_{0}\right)
$$

The contact deflections between the ball and the outer and the inner races are respectively denoted by $\delta_{1}$ and $\delta_{2}$. They can be written as

$$
\begin{gathered}
\delta_{1}=-v_{z} \cos \mu_{0}+v_{r} \sin \mu_{0}-\tilde{J} \\
\delta_{2}=-\left(u_{z}-v_{z}\right) \cos \left(\mu_{0}-u_{\theta}\right)+\left(u_{r}-v_{r}\right) \sin \left(\mu_{0}-u_{\theta}\right)-\tilde{J}
\end{gathered}
$$

The two races (outer and inner) exert forces $Q_{i}$ on the rolling element estimated by the Hertz theory:

$$
Q_{i}\left(v_{r}, v_{z}, v_{\theta}\right)=C_{e p}\left(\delta_{i}\right)^{3 / 2}
$$

(i $=1,2 ; 1$ - outer and 2 - inner)

The equilibrium equations in the radial frame $R_{3}$ are written as

$$
\begin{aligned}
& F_{r}\left(v_{r}, v_{z}, v_{\theta}\right)=F_{r}^{\text {Outer Race }}+F_{r}^{\text {Inner Race }}=-Q_{1} \sin \mu_{0}+Q_{2} \sin \left(\mu_{0}-u_{\theta}\right) \\
& F_{z}\left(v_{r}, v_{z}, v_{\theta}\right)=F_{z}^{\text {Outer Race }}+F_{z}^{\text {Inner Race }}=Q_{1} \cos \mu_{0}-Q_{2} \cos \left(\mu_{0}-u_{\theta}\right) \\
& M_{\theta}\left(v_{r}, v_{z}, v_{\theta}\right)=M_{\theta}^{\text {Outer Race }}+M_{\theta}^{\text {Inner Race }}=0
\end{aligned}
$$

\section{NUMERICAL RESULTS}

A radial displacement is applied on the inner race. We will show next, the influence of the contact angle $\alpha$ variation and the radial inner race displacement $u_{r}$ variation on the stiffness terms $K_{x x}, K_{y y}, K_{z z}, C_{x x}$ and $C_{y y}$.

\subsection{Influence of the contact angle variation}

The variation of the contact angle (between 30 and 40 degrees) and the angular position of the rolling element (between 0 and 360 degrees) are studied here. In figures 7, 8 and 9 we show the evolution of the axial stiffness $K_{x x}, K_{y y}$ and $K_{z z}$ respectively of the developed element. However, in figures 10 and 11, we present the evolution of the angular stiffness $C_{x x}$ and $C_{y y}$ respectively of the developed element.

We note that if the angular position is constant, the stiffness $K_{x x}$ and $K_{y y}$ increase with the contact angle (Fig. 7 and Fig. 8), while the stiffness $K_{z z}$ decrease with the contact angle (Fig. 9). The angular stiffness $C_{x x}$ and $C_{y y}$ are nulls for a contact angle 35 degrees (Fig. 10 and Fig. 11).

For a constant contact angle, the stiffnesses $K_{x x}, K_{y y}, C_{x x}$ and $C_{y y}$ have a sinusoidal behaviour while the stiffness $K_{z z}$ is constant. 
Stiffness $K_{x x}(\mathrm{MN} / \mathrm{m})$

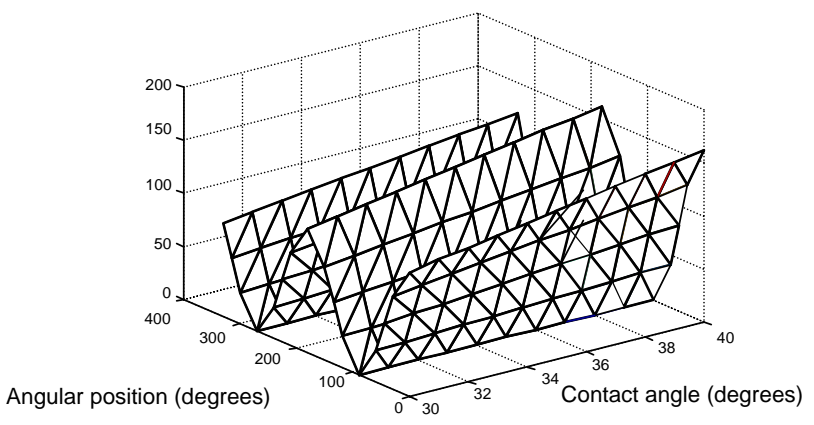

Figure 7: Stiffness $K_{x x}=K_{x x}(\phi, \alpha)$.

stiffness $K_{y y}(\mathrm{MN} / \mathrm{m})$

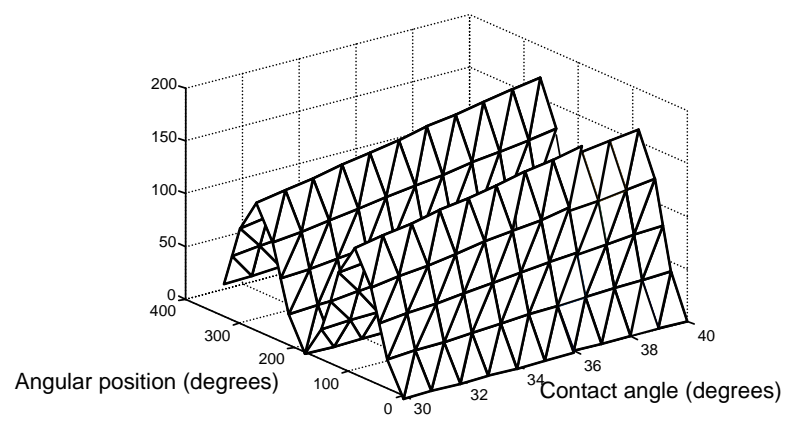

Figure 8: Stiffness $K_{y y}=K_{y y}(\phi, \alpha)$.

stiffness $K_{z z}(\mathrm{MN} / \mathrm{m})$

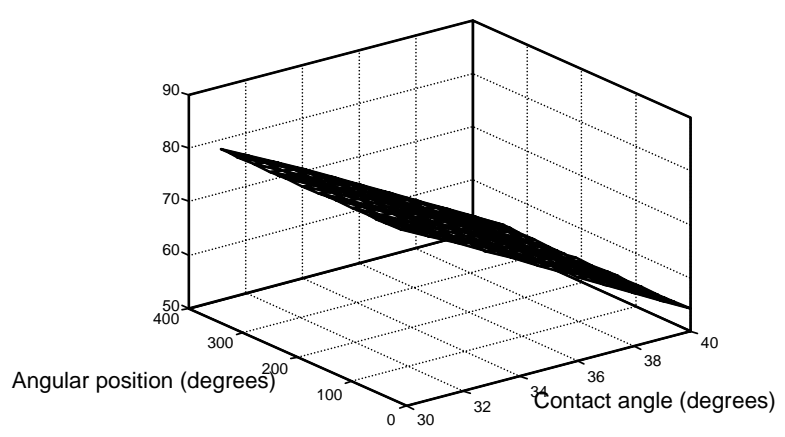

Figure 9: Stiffness $K_{z z}=K_{z z}(\phi, \alpha)$. 


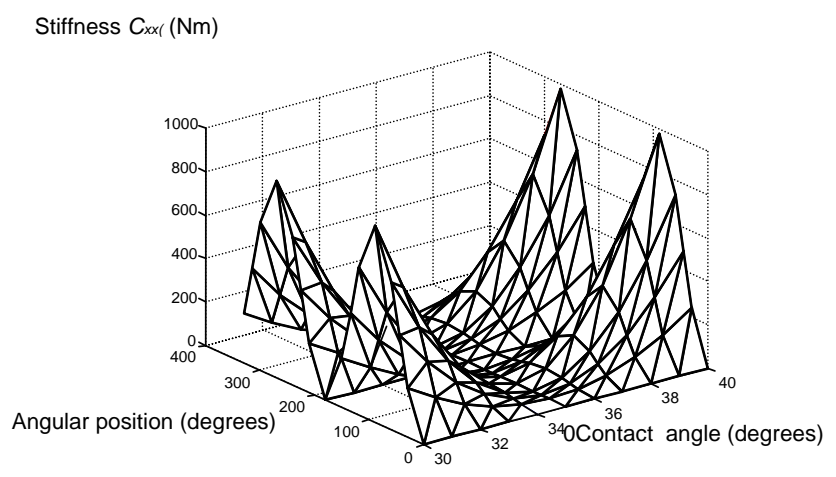

Figure 10: Stiffness $C_{x x}=C_{x x}(\phi, \alpha)$.

Stiffness $C_{y y}(\mathrm{Nm})$

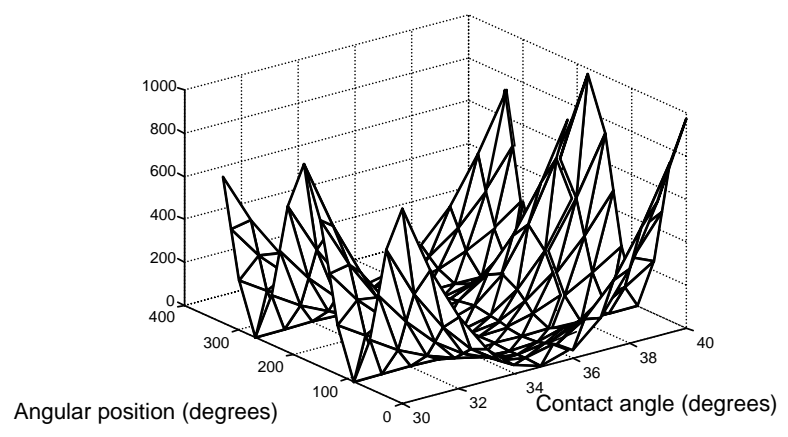

Figure 11: Stiffness $C_{y y}=\mathrm{C}_{y y}(\phi, \alpha)$.

\subsection{Influence of the inner race displacement $u_{r}$ variation}

We change the radial displacement between $10^{-4}$ to $10^{-3} \mathrm{~m}$. In figures 12,13 and 14 we show the evolution of the stiffness terms $K_{x x}, K_{z z}$ and $C_{x x}$ respectively of the developed element. We note that the stiffness terms $K_{x x}, K_{z z}$ and $C_{x x}$ increase non linearly according to the displacement. In fact this result is due to the non linear behaviour of the displacement (Hertz theory).

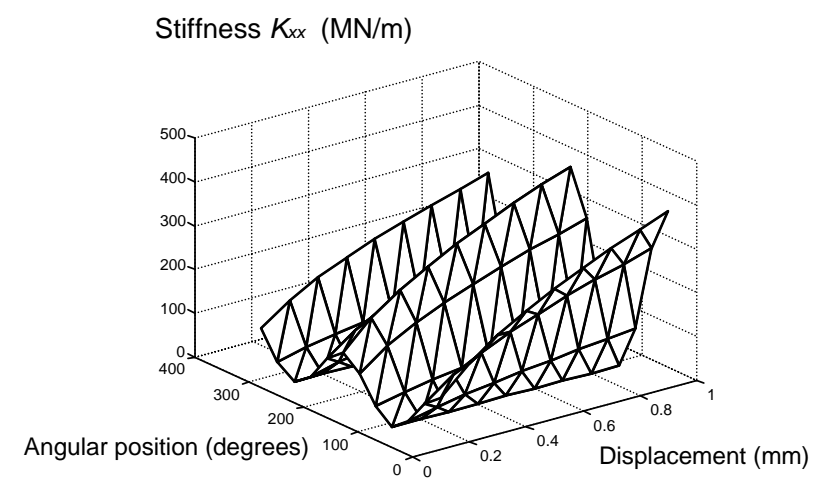

Figure 12: Stiffness $K_{x x}=K_{x x}\left(\phi, u_{r}\right)$. 


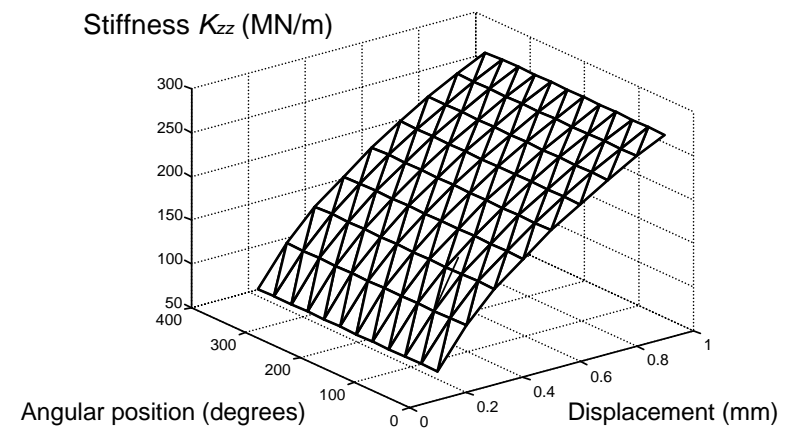

Figure 13: Stiffness $K_{z z}=K_{z z}\left(\phi, u_{r}\right)$.

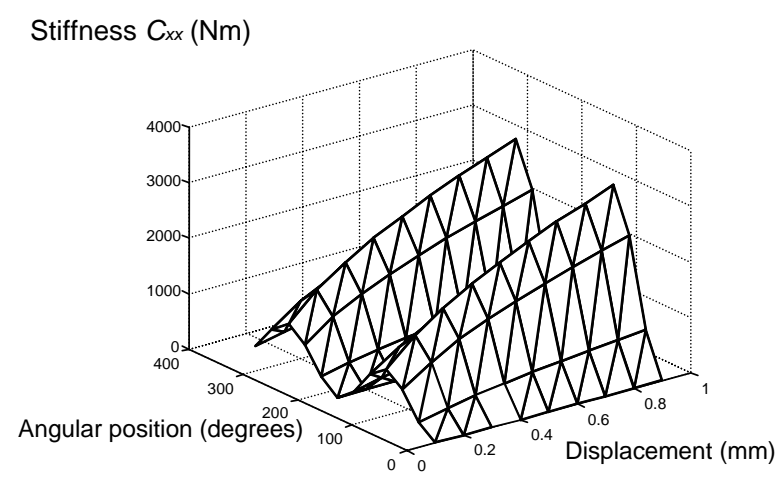

Figure 14: Stiffness $C_{x x}=\mathrm{C}_{x x}\left(\phi, u_{r}\right)$.

\subsection{Coupling between the stiffness matrix terms}

For a rolling element having an angular position $0^{\circ}$ and a radial displacement $10^{-4} \mathrm{~m}$, the tangent stiffness matrix is given by the table I.

Table I: Tangent stiffness matrix.

\begin{tabular}{|c|c|c|c|c|}
\hline $2.34110^{8}$ & 0 & $-1.96410^{8}$ & 0 & $6.5110^{5}$ \\
\hline 0 & 0 & 0 & 0 & 0 \\
\hline$-1.96410^{8}$ & 0 & $1.64810^{8}$ & 0 & $-5.4710^{5}$ \\
\hline 0 & 0 & 0 & 0 & 0 \\
\hline $6.5110^{5}$ & 0 & $-5.4710^{5}$ & 0 & $1.8110^{3}$ \\
\hline
\end{tabular}

In order to model the rolling element behaviour, we look for the eigenvalue and the eigenvecteor of the stiffness matrix:

$$
\begin{aligned}
& {\left[K_{T}^{e}\right]=\operatorname{diag}\left(3.9910^{8}, 0,0,0,0\right)} \\
& {\overrightarrow{e_{X}}}^{T}=\left\{\begin{array}{lllll}
0.76 & 0 & -0.64 & 0 & 0
\end{array}\right\}_{R_{1}}
\end{aligned}
$$

We find that the rolling element can be modelled by a spring having stiffness $3.9910^{8} \mathrm{~N} / \mathrm{m}$ in the direction $\overrightarrow{e_{X}}$ (the rotations around $\overrightarrow{e_{x}}$ and $\overrightarrow{e_{y}}$ are neglected). 
This result shows the importance of this study: a roller bearing can not be modelled by the association of three springs in the directions $\overrightarrow{e_{x}}, \overrightarrow{e_{y}}$ and $\overrightarrow{e_{z}}$. The coupling between these springs seems to be very important, and necessitate using a finite element "rolling element".

\section{CONCLUSION}

It was developed a finite element having two nodes and six degrees of freedom per node. The stiffness matrix of this element is deduced from the non-linear equilibrium of the rolling element. The Newton Raphson method is used to solve those equilibrium equations. Three numerical analysis are given in order to show the influence of the variation of some parameters on the stiffness matrix terms.

This analysis shows the presence of the important coupling between the stiffness terms. This coupling can reach $96 \%$ in the unfavourable case (rolling element situated in an angular position $30^{\circ}$ ). The bearing can not be modelled by linear springs association along three orthogonal directions.

The global bearing stiffness matrix can be computed by assembling all rolling element stiffness matrixes. This matrix is then introduced in a global model defining the system stiffness.

\section{REFERENCES}

[1] Jones, A. B. (1960). A general theory for elastically constrained ball and radial roller bearings under arbitrary load and speed conditions, Transactions of the American Society of Mechanical Engineers, Journal of Basic Engineering, Vol. 82, 309-320

[2] Palmgren, A. (1959). Ball and roller bearings engineering, Burbank, Philadelphia

[3] While, M. F. (1979). Rolling element bearing vibration transfer characteristics: effects of stiffness, Transactions of the American Society of Mechanical Engineers, Journal of Applied Mechanics, Vol. 46, 677-684

[4] Krauss, J.; Blech, J. J.; Braun, S. G. (1987). In situ determination of roller bearing stiffness and damping by model analysis, Transactions of the American Society of Mechanical Engeneers, Journal of Vibration, Acoustics, Stress and Reliability in design, Vol. 109, 235-240

[5] Drago, R. J. (1980). How to Design Quiet Transmission, Machine Design, 175-181

[6] Lim, T. C.; Singh, R. (1990) Vibration transmission through rolling element bearings. Part I: Bearing stiffness formulation, Journal of Sound and Vibration, Vol. 139, No. 2, 179-199

[7] Demul, J. M.; Vree, J. M.; Maas, D. A. (1989). Equilibrium and associated load distribution in ball and roller bearing loaded in 5 degrees of freedom while neglecting frictions. Part II: Application to roller bearings in experimental verification, Transactions of the American Society of Mechanical Engineers, Journal of Tribology, Vol. 111, 149-155

[8] Bourdon, A. (1997). Modélisation dynamique globale des boites de vitesses automobile, thèse de doctorat, institut nationale des sciences appliquées de Lyon

[9] Lahmar, F. (2000). Interaction entre la dynamique de l'engrènement et les paliers à roulement", thèse de doctorat, Institut Nationale des Sciences Appliquées de Lyon

[10] Gupta P. K. (1984). Advanced Dynamics of Rolling Elements, Springer-Verlag, New York

[11] Nelias, D. (1999). Contribution à l'étude des roulements. Modélisation globale des roulements et avaries superficielles dans les contacts EHD pour des surfaces réelles ou indentées, dossier d'habilitation à diriger des recherches, Institut nationale des sciences appliquées de Lyon.

[12] Dhatt, G.; Touzot, G. (1984). Une présentation de la méthode des éléments finis. Maloine S.A. Editeur, Paris

[13] Stacke, L. E.; Friston, D., (1999). Simulation of rolling element bearings, SKF Nova AB, Göteborg, Sweden

[14] Hirotoshi, A. (1997). Rolling bearing analysis program package "BRAIN", Motion and Control Journal, No. 3, 15-24 